\title{
La fonction pragmatique de la ponctuation dans la rédaction professionnelle : une étude de cas
}

\author{
Romain, Christina, Rey, Véronique, \& Pereira, Marie-Emmanuelle \\ Aix Marseille Université - ESPE Aix-Marseille \\ Laboratoire Parole et Langage CNRS UMR 7309 \\ christina.romain@univ-amu.fr \\ Aix Marseille Université - ESPE Aix-Marseille \\ CREDO, CNRS UMR 7308 \\ veronique.rey-lafay@univ-amu.fr \\ Aix Marseille Université - ESPE Aix-Marseille \\ CREDO, CNRS UMR 7308 \\ veronique.rey-lafay@univ-amu.fr
}

\begin{abstract}
Résumé. La ponctuation est au centre de débats qui portent notamment sur sa(es) fonction(s). Bessonnat (1991:4) fait reposer l'analyse fonctionnelle de la ponctuation sur une fonction syntaxique, une fonction sémantique et une fonction communicationnelle. Anis (2004) met luimême l'accent sur la dimension énonciative de la ponctuation en tant que lieu d'investissement du scripteur. De même, Lapacherie (2014) rappelle que trois thèses ont été mises en avant au cours des derniers siècles : la corrélation à l'oral, l'information sémantique et la cohérence syntaxique. Thèses auxquelles s'ajoute, au cours des dernières décennies, celle de l'orientation pragmatique de la dimension énonciative des signes de ponctuation qui ne se réduisent pas alors aux guillemets ou autres tirets (Dahlet, 2003:147). Dans cette perspective d'anlayse, Narjoux souligne que « La ponctuation nous dit assurément beaucoup de celui qui choisit d'y recourir. » (2010:12) et prolonge son propos de la façon suivante : "Sans doute, la ponctuation dans son ensemble a-t-elle une dimension énonciative, plus ou marquée, et qui transparait jusque dans les choix de celui qui écrit pour tel ou tel signe, à tel ou tel endroit. " (2010:59). Nous proposons de nous positionner sur l'aspect communicationnel voire interactionnel de la ponctuation en faisant reposer notre analyse sur l'étude de pratiques discursives de rédacteurs professionnels (RP). Il s'agit de comprendre l'enjeu de la ponctuation dans sa corrélation avec d'autres indicateurs de relation interdiscursive convoqués par le RP pour produire un écrit visant une relation particulière avec son destinataire. Nous entendons donc la ponctuation comme la trace d'un positionnement discursif guidé par une intention de communication spécifique (Dahlet, 2003). Notre approche est résolument empirique et nous avons constitué pour cela un corpus comprenant des écrits pour le grand public rédigés par des personnes travaillant au sein d'un service juridique ou administratif qui ont donné lieu à une réécriture par des RP du fait du manque de lisibilité. L'analyse révèle une spécificité du rédacteur professionnel qui ajoute à une pratique normalisée de la ponctuation une dimension pragmatique. La ponctuation relèverait donc simultanément de la langue et de la communication en lien avec la relation interdiscursive que le scripteur souhaite mettre en place avec son lecteur. La ponctuation est notamment analysée comme un espace où se jouent des rapports de places et de faces et une certaine négociation. Notre but est de décrire comment les points de vue en confrontation peuvent influer sur la structuration et le choix de la ponctuation.
\end{abstract}

Abstract. The pragmatic function of punctuation in professional writing: a case study. We suggest to position ourselves on the communicative aspect of interactional punctuation by basing our analysis on the study of discursive practices of professional writers (R.P.). This includes the issue of punctuation in its correlation with other indicators of interdiscursive relationship summoned by R.P. to produce written to a special relationship with the receiver. 


\begin{abstract}
We consider the punctuation as the trace of a discursive positioning guided by an intention of specific communication (Dahlet, 2003). Our approach is empirical and we assembled a corpus of texts for the general public written by people working in legal or administrative service, and which resulted in a rewrite by R.P. due to massive comprehension difficulties invoked by the adressees. We propose to analyze the punctuation as a space where places are and reports play faces and some negotiation. Our goal is to describe how the confrontation in views can influence the structuring and choice of punctuation.
\end{abstract}

Dans le cadre de cet article, nous proposons de nous positionner sur l'aspect communicationnel voire interactionnel de la ponctuation en analysant des pratiques discursives de rédacteurs professionnels (R.P.). La recherche actuelle montre que le savoir-faire en écriture du R.P. requiert des compétences dans le code écrit mais relève aussi de compétences en écriture de haut niveau et de compétences de communication (Beaudet : 2010 ; Clerc \& Beaudet : 2008 ; Labasse : 2008). Nous nous inscrivons dans la lignée des travaux confrontant la pratique de la rédaction professionnelle à son «efficacité interactionnelle »(Clerc \& Beaudet, 2008). Notre recherche, commencée il y a deux ans, vise à identifier les éléments caractéristiques de l'écriture professionnelle (Romain, Pereira et Rey, 2015ab ; Pereira, Romain et Rey, 2015) : après avoir étudié les choix linguistiques permettant l'élaboration d'une relation interpersonnelle et interdiscursive spécifique, nous nous intéressons à la fonction pragmatique de la ponctuation. Cet article vise à comprendre l'enjeu de la ponctuation dans sa corrélation avec d'autres indicateurs de relation interdiscursive convoqués par le R.P. pour produire un écrit visant une relation particulière avec son destinataire. Nous entendons donc la ponctuation comme la trace d'un positionnement discursif guidé par une intention de communication spécifique (Dahlet, 2003).

Pour appréhender cet enjeu, nous tenterons de répondre aux questions suivantes : Comment celui qui est réputé avoir une expertise dans le code écrit utilise-t-il la ponctuation ? Comment inclut-il dans la représentation qu'il se fait de cet outil la relation avec son lecteur à venir ? Quelle(s) fonction(s) de la ponctuation pour un rédacteur professionnel? Quelle(s) perception(s) ? Quelle conscience de la ponctuation (cf. travaux de Siouffi) liée aux enjeux interactionnels de l'écrit commandé ? Quel rôle en lien avec la relation interpersonnelle et la relation interdiscursive mises en place par le R.P. ? Comment ce fait de langue devient un fait de relation sociale par les choix opérés dans le positionnement relationnel ?

\title{
1 La ponctuation ne se réduit pas à un guidage de la lecture/oralisation du texte
}

Dans les années 1980, les travaux en linguistique de Nina Catach et de son équipe HESO, mais aussi ceux de Michel Fayol en psychologie cognitive, ont érigé la ponctuation en un champ de recherche scientifique (pour une mise en perspective historique détaillée voir Saint-Gérand, 2000).

Dès 1980, Catach (1980) évoquait la valeur idéographique des signes de ponctuation en tant que signes porteurs de sens et insistait sur leur lien avec un système spécifique de communication («système second de communication » (1980: 16) à côté de celui du langage lui-même). Elle soulignait alors l'importance dans les recherches futures de s'atteler à l'analyse systématique de toutes les réalisations de ces signes et 
de «mieux démontrer que finalement il ne s'agit pas d'éléments auxiliaires, non indispensables au langage, mais, au contraire, d'une acquisition historique fondamentale de la communication humaine » (1980 : 27). Plus spécifiquement, Perrot (1980 : 67-76), dans le même numéro de Langue Française, étudiant les fonctions linguistiques de la ponctuation, insistait sur trois types de signalisation relevant de la ponctuation : «la relation du locuteur avec le contenu de son discours », "l'organisation des rapports interpersonnels de la communication » et «l'organisation du texte comme constituant l'ensemble d'un « dire » ». A la fin des années 1980, Védénina (1989: 144) soulignait que l'intonation et la ponctuation sont des formes différenciées d'un même signifiant. En effet, chacune « dans son domaine, est porteuse d'indications syntaxiques, thématiques et énonciatives ; quand nous lisons un texte, nous décodons les topogrammes et si nous oralisons, nous utilisons des marques intonatives correspondantes » (Anis, 1988 : 154-155). Passerault (1991 : 90-91) proposait quant à lui trois approches de la ponctuation dans le cadre de la production d'écrit :

- La ponctuation comme constitutive d' « une trace des processus de linéarisation ». Cette approche consiste « à rechercher une correspondance entre la nature des marques de ponctuation utilisées dans le texte et l'organisation de la représentation préalable à la mise en texte ».

- La ponctuation comme la trace de

\begin{abstract}
« la manière dont le sujet structure son texte en parties, l'adaptant aux besoins du lecteur ; elle est alors considérée comme la trace des processus de planification (Bronckart et al., 1985 ; Schneuwly, 1984) étant considérés comme moins dépendants de la représentation des contenus en mémoire que des modèles langagiers (superstructuraux) instanciés sur la base du traitement des paramètres de la situation (but, destinataire...). Cette seconde approche consiste donc à rechercher dans quelle mesure, à partir d'un certain niveau de pratique de l'écrit, la ponctuation peut relever d'une activité de structuration du texte liée au contexte plutôt qu'à l'organisation macrostructurale des contenus de la représentation. »
\end{abstract}

- La ponctuation comme le

«lieu privilégié d'accomplissement de l'activité de planification, le terme de planification étant entendu d'une manière un peu plus différente de la conception précédente (cf. par exemple Hayes et Flower, 1980 ; Van Dijk et Kintsch, 1983). La planification désigne ici les processus par lesquels le sujet élabore des plans, de différents niveaux, préalablement à la mise en texte proprement dite. Cette troisième approche consiste à analyser les paramètres temporels (temps de pause), aux différents lieux de ponctuation, comme indicateurs «des ressources cognitives» nécessaires à la planification. »

On le voit, très rapidement, les chercheurs se sont accordés à envisager la ponctuation comme un phénomène qui ne se réduit ni à la diction elle-même, ni à l'accompagnement de l'oralisation du texte. C'est cette perspective d'étude qui est la nôtre puisque nous avons cherché à nous éloigner de l'étude du rôle strictement phrastique de la ponctuation pour mieux interroger son rôle énonciativo-pragmatique.

\title{
2 Du rôle phrastique au rôle énonciativo-pragmatique de la ponctuation
}

Tournier (1980 : 32) concédait l'« analogie entre la ponctuation de l'écrit et la prosodie de l'oral : la première permet une bonne compréhension du texte, qui permet ensuite au lecteur de réaliser judicieusement pauses et intonations » et Arrivé et al. (1986 : 536) soulignaient que «L'écrit se caractérise [...] par le fait qu'il donne une manifestation segmentale à ce qui est marqué, à l'oral, par des éléments suprasegmentaux. ». Dix ans après la parution du numéro 45 de Langue Française de 1980, Drillon (1991 : 82-88) insiste quant à lui sur la non correspondance entre la ponctuation et la diction et sur le caractère écrit de la ponctuation. Plus spécifiquement, dans le numéro de l'Information Grammaticale consacrée à la ponctuation, Pétillon (2004 : 4), dans l'introduction du numéro, relève 


\begin{abstract}
« la remarquable plasticité des signes de ponctuation : plongés dans la structuration du texte ou affleurant seulement son épiderme ; oscillant entre les contraintes grammaticales et l'inédit scripturaire, "lieux aigus du passage de la langue au discours » (S. Pétillon), ils constituent, de façon manifeste, une gamme de signes privilégiés pour l'investissement énonciatif des sujets écrivants. ».
\end{abstract}

Anis (2004), dans le même numéro, met l'accent sur la dimension énonciative de la ponctuation en tant que lieu d'investissement du scripteur. Enfin, Lapacherie (2014), qui s'interroge sur ce qu'indiquent les signes de ponctuation, rappelle que trois thèses se sont affrontées ou ont au contraire cohabité au cours des derniers siècles : la corrélation à l'oral, l'information sémantique et la cohérence syntaxique. Thèses auxquelles s'ajoute, au cours des dernières décennies, celle de l'orientation pragmatique de la dimension énonciative des signes de ponctuation qui ne se réduisent pas alors aux guillemets ou autres tirets (cf. Dahlet, 2003 : 147). Dans une étude sur le rôle de la ponctuation dans un corpus épistolaire (écritureordinaire), Jagueneau conclut de la façon suivante :

«Dans ce texte épistolaire, au-delà des fonctions spécifiques de chaque ponctuant, le trait essentiel est sans doute la rareté de la ponctuation interne à l'alinéa, et sa conséquence, la mise en évidence de la disposition en blocs réguliers, est d'autant plus frappante que la soulignent, entre les alinéas, le point, les blancs de fin et de début de ligne et la majuscule. De l'allocution initiale à la signature, la lettre se dessine alors comme le combat contre le vide du silence qui renait à chaque point «final » d'alinéa dans l'attente de l'alinéa à venir, et que le déroulement du fil continu du paragraphe suivant efface, avant de lui laisser définitivement la place. Sans point final » (2014).

Neveu (2014) illustre parmi les marqueurs les plus courants :

« (i) les faits de modulation (c'est-à-dire les ajouts typographiques : italique, gras, soulignement, guillemets, et les divers procédés d'emphase graphique comme les signes ponctuants de l'affectivité), (ii) les faits de segmentation (c'est-à-dire ceux qui sont engagés dans les mécanismes de hiérarchisation des zones de localité et qui forment des frontières graphiques intraphrastiques ou transphrastiques : ponctuation de détachement et de clôture des segments syntaxiques, modes d'insertion des séquences textuelles dans les structures englobantes, titres, types de pans numériques, alphanumériques, etc. -, numérotation et structure volumétrique des paragraphes, gestion des alinéas et des espaces, etc.). »

Il poursuit en créditant

\begin{abstract}
«l'encodeur d'une capacité d'initiative plus importante que ne le laissent entendre les analyses développant la thèse d'un modèle normatif hégémonique de démarcation dans la réalisation écrite de la langue. Les études de corpus font ressortir que les « incohérences » de la ponctuation sont fréquemment le fait d'une approche grammaticale et non pas discursive du problème. A l'écrit, l'encodage ne peut être borné à l'observance aveugle des conventions graphiques. Le scénario plaisant d'un scripteur inhibé et immature, trempant sa plume dans l'encrier de l'ordre social, et écrivant sous la dictée du législateur graphique, a vu récemment le nombre de ses amateurs sensiblement diminuer sous l'influence de la communication multimédia et du développement de l'écrit d'écran. »
\end{abstract}

On observe ici l'évolution de l'analyse de la ponctuation d'une ponctuation phrastique à une ponctuation textuelle où le scripteur fait des choix certes sémantiques ou encore syntaxiques mais aussi et surtout pragmatiques... Fayol et Abdi (1988) ont ainsi conduit une expérience concernant la nature et la fréquence des différents signes de ponctuation de support textuels, plus exactement de scripts en ce qu'ils présentaient l'avantage de reposer sur des séquences événementielles suffisamment rigides quant à leur organisation à la fois séquentielle et hiérarchique. A l'issue de l'analyse de leur corpus, les chercheurs ont établi une corrélation entre « la force de la ponctuation » et « le degré de liaison des propositions dans l'organisation du script». Passerault, mentionnant cette étude (1991 : 91), souligne que 
«les ponctuations sont plus fortes lorsqu'elles délimitent des propositions correspondant à des épisodes différents, elles sont plus faibles lorsqu'elles délimitent des propositions fortement reliées entre elles dans la représentation. Cette relation apparaît aussi bien chez les élèves de sixième que chez les adultes. ».

Conduisant une étude similaire (1991: 91), il conclut pour sa part que

« la ponctuation est utilisée pour marquer les relations qu'entretiennent les éléments au niveau de l'organisation de la représentation pré-linguistique. Ce qu'on observe ici pour les scripts est parfaitement conforme aux observations de Fayol et Lété (1987) mentionnées plus haut concernant les récits ».

On comprend pourquoi la ponctuation revêt différentes fonctions et ne peut être limitée à une seule. Ainsi, Bessonnat (1991 : 4), après avoir décrit la fonction principale de la ponctuation comme la régulation de la « contradiction entre le code et le discours », fait reposer son analyse fonctionnelle sur trois fonctions : la fonction syntaxique (communément admise), la fonction communicationnelle (cf. les travaux de Védénina et Anis) et la fonction sémantique (cf. les travaux de Fonagy). Jaffré (1991 : 61-83) revient sur ces trois fonctions en les décrivant. La fonction syntaxique «consiste à délimiter des segments à l'intérieur d'un énoncé («démarcation»)» (ibid: 71), la fonction communicationnelle ou énonciative correspond aux

\begin{abstract}
«diverses contraintes dictées par les finalités du message. C'est le cas de la thématisation [...] C'est également le cas des incursions diverses de l'auteur, en fonction de son affectivité ou de son jugement ou encore de l'insertion de segments textuels en provenance de textes extérieurs (citation, proverbe, discours direct, etc.) » (ibid : 74)1,
\end{abstract}

et la fonction sémantique pour laquelle il rappelle que
«Chez Védénina, les aspects syntaxique et « communicatif de la ponctuation ont bien à voir avec le sens, mais d'une manière indirecte qui vise à satisfaire les besoins de l'expression linguistique. Un autre aspect, proprement «sémantique» celui-là, envisage une corrélation directe entre ponctuation et sens. Dans ce domaine, Védénina fait une différence entre signes de démarcation, signes de régulation et signes de qualification. » (ibid: 78)2

De même, selon Narjoux (2010), la ponctuation participe à la structuration organisationnelle du texte en produisant des informations prosodiques (ex : «Il est arrivé... »vs «Il est arrivé !»), des relations syntaxiques » (ex : «Le renard, terrifiant et affamé, dévora la poule. »), des effets d'ordre sémantique (ex : «Les élèves qui ont des bonnes notes iront en sortie la semaine prochaine. » $v s$ « Les élèves, qui ont des bonnes notes, iront en sortie la semaine prochaine. »), et en notant des informations communicationnelles relevant des marqueurs du discours rapporté (ex : «J'en suis ravie ! dit-elle en souriant. »). Plus spécifiquement, Narjoux prend position en affirmant que

\begin{abstract}
«Certains des signes de ponctuation ont une fonction première énonciative, c'est-àdire qu'ils nous renseignent sur des changements dans la situation d'énonciation (qui parle ? à qui ? dans quel état d'esprit ?). Ils signalent la présence du locuteur (le point d'interrogation ou d'exclamation, de suspension, voire l'astérisque), un changement de locuteur (le tiret, les guillemets), le discours rapporté ou un décrochage énonciatif (les parenthèses et les tirets doubles, les crochets, les guillemets et l'italique permettent de signaler la citation de discours réels ou virtuels, des insertions de commentaires, de mettre en valeur certains segments pour le lecteur ou, comme l'astérisque, un appel de note). En somme ils structurent le discours en différents plans énonciatifs, et signalent les différentes voix qui le composent ; ils révèlent la complexité de la trame qui forme tout discours. » (2010:58).
\end{abstract}

Mais Narjoux complète encore son propos en rangeant dans les signes à fonction énonciative ceux signalant la présence de l'énonciateur et qui sont de fait susceptibles d'informer sur le rapport de ce 
dernier avec autrui (elle nomme ainsi le point d'exclamation et les points de suspension notamment) ${ }^{3}$. Cette auteure souligne surtout que
«La ponctuation nous dit assurément beaucoup de celui qui choisit d'y recourir. » (2010 : 12) et prolonge son propos de la façon suivante : «Sans doute, la ponctuation dans son ensemble a-t-elle une dimension énonciative, plus ou moins marquée, et qui transparait jusque dans les choix de celui qui écrit pour tel ou tel signe, à tel ou tel endroit. » $(2010: 59)$.

Dans une étude spécifique des liens entre ponctuation et énonciation, Dahlet (2003 : 124) souligne la congruence nécessaire ou recherchée entre le sens et la ponctuation (qu'elle décrit comme un " opérateur syntaxico-sémantique et énonciatif »). Elle distingue le « sens référé », qui ne demande au lecteur que des compétences linguistiques, et le «sens visé », qui repose sur l'intention de communication. Elle sépare donc ce qui relève de la norme (pré-fixée à l'utilisation des signes), de la clarté et de la visibilité (en tant que facilitatrice du traitement de l'information) mais aussi et surtout de l'intention de communication qu'elle décrit comme rendant compte « des modalités d'interprétation telles que le scripteur chercher à les préfigurer. » Dahlet insiste sur le fait que ces paramètres dépendent d'un choix à la fois méthodologique et expérimental permettant de mettre en avant, d'identifier, ce qui relève du système de la ponctuation (fixe, pré-établi, visant le traitement informatif du contenu écrit) et ce qui relève de l'activité du sujet scripteur lui-même et qui vise sa relation avec autrui. C'est ce dernier aspect de la question qui retient toute notre attention dans l'analyse de notre corpus.

Dahlet (2003 : 25-26) décrit le corpus de la ponctuation en distinguant la ponctuation de mot (point abréviatif, parenthèses, points de suspension, trait d'union, apostrophe) de la ponctuation de phrase (s'inspirant de la classification proposée par Anis, 1988) et en différenciant pour cette dernière les signes de séquences (alinéa, point, point virgule, virgule) des signes d'énonciation hiérarchiseurs discursifs (les deux points, les parenthèses, le tiret double), les marqueurs expressifs (capitale, soulignement, italique, gras, tiret), les marqueurs interactifs (point d'interrogation, point d'exclamation, points de suspension), le discours cité (guillemets, italique, tiret de dialogue).

Quant à nous, à travers notre analyse, nous étudierons le choix global (macro) de la ponctuation. Autrement dit, nous questionnerons la ponctuation du texte écrit dans sa perspective d'entrée en relation avec le scripteur : quel choix de ponctuation (en lien avec d'autres indicateurs tels que les procédés d'interpellation et de positionnement, la répétition, le champ lexical, les choix syntaxiques, etc.) en fonction d'une relation coopératrice/négociatrice $v s$ agonale ?

\section{Notre problématique}

Notre étude de la ponctuation est résolument empirique et nous avons constitué pour cela un corpus comprenant des écrits pour le grand public rédigés par des personnes travaillant au sein d'un service juridique ou administratif (corpus $\mathrm{A}$ : formulaire 1 de réclamation Hyundai ; règlement intérieur du service accueil de la petite enfance d'une mairie du sud-est de la France) qui ont donné lieu à une réécriture par des R.P. du fait du manque de lisibilité (difficultés massives de compréhension invoquées par les destinataires) (corpus B : formulaire 2 de réclamation Hyundai ; livret d'accueil municipal de la petite enfance qui vise à transmettre les informations du règlement intérieur).

Nous émettons ici l'hypothèse que la ponctuation, dont il a déjà été démontré qu'elle était formée à la fois d'un signifiant et d'un signifié (Tournier : 1980), fonctionnerait, en dépendance des choix du scripteur et des moments voire des séquences textuelles, comme des actes de langage présentant un effet perlocutoire spécifique (coopération vs conflit). C'est dans cette perspective d'analyse que nous nous inscrivons en questionnant la ponctuation dans sa perspective textuelle et dans sa dimension énonciativo-pragmatique. Nous envisageons les signes de ponctuation à partir de la notion d'hétérogénéité : marques de la relation interpersonnelle à travers celles de la relation interdiscursive et rapport du sujet de l'écriture au réel et à la langue, à lui-même (Bikialo, 2004). C'est donc la dimension pragmatique de la ponctuation (son choix en termes de relation interdiscursive spécifique) qui retient ici toute notre attention. Le propos est de 
regarder, chez le scripteur, les éléments marquant les façons de se représenter et d'entrer en contact avec le lecteur. Il s'agit d'une certaine décentration du sujet-scripteur, au profit d'un acte de co-énonciation, une « mise à distance » pour gérer au mieux la visée pragmatique ; cette ponctuation-là à l'échelle du texte, refléterait cette représentation de l'autre en train de lire.

\section{Analyse du corpus}

Nous allons comparer la réécriture des documents initiaux avec leurs réécritures totales ou partielles. Pour chaque document, (formulaire de réclamation et règlement intérieur/livret d'accueil), nous avons choisi deux extraits. Nous questionnerons les changements opérés en termes de ponctuation et nous prendrons nécessairement en compte les modifications d'ordre syntaxique et les enjeux relationnels de cette réécriture.

\subsection{Réécriture d'un formulaire de réclamation}

\subsubsection{Extrait 1}

Extrait avant réécriture (formulaire 1)

POUR RECEVOIR UNE COMPENSATION, VOUS DEVEZ ÊTRE ADMISSIBLE À TITRE DE RECLAMANT. SI VOUS ÊTES ADMISSIBLE À TITRE DE RÉCLAMANT, LE FORMULAIRE DE RÉCLAMATION COMPLÉTÉ DE MÊME QUE LES PIÈCES JUSTIFICATIVES DOIVENT AVOIR ÉTÉ REÇUS PAR LE GESTIONNAIRE DES RÉCLAMATIONS AU PLUS TARD LE 12 DÉCEMBRE 2005

\section{FORMULAIRE DE RÉCLAMATION}

Il est possible que vous soyez admissible à titre de réclamant en vertu de cet accord de règlement si, en date du 9 septembre 2002, vous étiez propriétaire ou locataire d'un des véhicules Hyundai suivants : Accent. Elantra, Santa Fee, Sonata, Tiburon ou XG300. Pour déterminer si vous êtes admissible à titre de réclamant, référez-vous à l'Étape 1 (ci-dessous). Pour plus d'information à l'égard de la compensation, veuillez consulter le Tableau A.

LES FORMULAIRES DE RÉCLAMATION DÛMENT COMPLÉTÉS DOIVENT ÊTRE TRANSMIS À HYUNDAI AUTO CANADA, A TITRE DE GESTIONNAIRE DES RÉCLAMATIONS, À L'ADRESSE SUIVANTE :

Hyundai Auto Canada,

75 Frontenac Drive,

Markham, Ontario

L3R 6H2

À l'attention de: Customer relations (Horsepower Seulement)

Extrait après réécriture (formulaire 2)

Recours collectif contre Hyundai Canada

Formulaire de réclamation

À retourner par la poste au plus tard le 12 décembre 2005. 
En remplissant ce formulaire de réclamation, vous nous permettrez d'obtenir toute l'information nécessaire pour vous rembourser, si c'est le cas. Les sections 1 et 2 vous permettront de savoir si vous êtes admissible à une réclamation. Si vous êtes admissible, vous devrez répondre ensuite à toutes les sections du formulaire.

Les instructions pour nous faire parvenir ce formulaire se trouvent à la section 12 .

\subsubsection{Le titre, les sur- et sous-titres}

\section{Le titre}

Si dans le formulaire 1 le titre est mis en gras et en majuscules, il perd les majuscules dans le second formulaire tout en restant en gras.

\section{Les sur- et sous-titres}

Formulaire 1 : on observe la présence d'un paragraphe en majuscules et en gras au-dessus du titre. Il est constitué de deux phrases complexes comportant chacune un circonstant détaché en début de phrase par une virgule. Ce paragraphe est compact et sa visibilité est peu performante : les interlignes sont minimaux et le texte est centré. Les phrases sont longues, elles initient et développent déjà l'information qui doit être donnée dans le contenu du texte : le lecteur est immédiatement engagé dans l'action. Ainsi constate-t-on une relation de sur-information par rapport à sa localisation du paragraphe, c'est-à-dire par rapport à sa position de descripteur du titre introductif du formulaire. L'énonciateur, la firme Hyundai, est dans une position haute et menaçante de communication. Cette relation génère une menace pour la face du destinataire qui est enjoint, avant même l'entrée dans le corps du formulaire, de comprendre ce qui sera expliqué plus tard, de mémoriser ces informations et les injonctions explicites et implicites en découlant.

Formulaire 2 : le paragraphe du formulaire 1 est remplacé par un sur-titre (identification de la nature du document) et par un sous-titre (date de retour en gras du document dûment rempli). Ces deux informations essentielles sont données sous forme nominale permettant de saisir immédiatement les mots clefs afin d'en constituer le sens. La police est plus petite que celle du titre lui-même, ce qui permet de graduer l'importance des informations.

Seules les informations clefs sont mises en avant dans le souci d'une relation d'information. Il s'agit donc d'une autre procédure de construction de la relation avec le lecteur : la relation est immédiatement coopératrice voire négociatrice de la part de la firme (position haute et coopératrice).

La réécriture a consisté ici à alléger les informations, à les simplifier, à les rendre visibles mais aussi lisibles. Les titres visent à générer deux comportements différents chez le lecteur et cela a un effet sur la ponctuation puisque dans le formulaire 1 il y a une surcharge de ponctuation alors que dans le formulaire 2 celle-ci se fait plus discrète.

\subsubsection{Introduction du formulaire}

Formulaire 1: il est formé de deux paragraphes et d'un encadré indiquant l'adresse où retourner le formulaire. Si le premier paragraphe contient trois phrases, le second en comprend une seule et est écrit en majuscules.

\section{Le premier paragraphe :}

- phrase 1 : deux virgules encadrant un circonstant (informant sur la date) et deux points introduisant une énumération comprenant 4 virgules ;

- phrase 2 : une virgule isolant le circonstant en début de phrase ;

- phrase 3 : une virgule séparant le circonstant du reste de la phrase dans une position initiale à nouveau.

Le second paragraphe : une phrase unique en majuscules avec un groupe détaché explicatif entre deux virgules. 
Le troisième paragraphe : adresse encadrée.

Formulaire 2 : cette réécriture est formée d'un seul paragraphe constitué de 4 phrases.

- phrase 1 : deux virgules (isolant les circonstants en début et en fin de phrase);

- phrase 2 : pas de ponctuation intra-phrastique ;

- phrase 3 : une virgule en introduction isolant un circonstant ;

- phrase 4 : pas de ponctuation intra-phrastique.

La réécriture de cette introduction montre le souci du R.P. de présenter cette introduction sous la forme d'un paragraphe unique, fonctionnant comme un tout homogène, au sein duquel, il fait alterner, à deux reprises, une phrase isolant des circonstants par une virgule et une phrase sans ponctuation intraphrastique.

Dans la rédaction initiale, on est en présence d'un effet de sur-énonciation « où on ordonne de faire, on enjoint de faire ... » et non d'un effet de co-énonciation où « on dit des choses », « on parle à... » comme dans la réécriture. Ce second formulaire dénote une volonté de clarté (lecture plus fluide, mieux guidée formellement) et de visibilité (compréhension des informations = coopération). On «progresse dans les informations avec des pauses en fonction des phrases. »

Ici il n'y a pas de suppression de phrase mais une simplification des contenus, avec une réorganisation de la distribution des compléments convoquant alternativement une virgule ou une absence de ponctuation intra-phrastique.

Dans ce premier extrait, si la simplification syntaxique génère une simplification de la ponctuation, la modification de la structure énonciative agit elle aussi sur la structure syntaxique et sur la ponctuation. La ponctuation se situant à la fois dans la structure syntaxique et dans la structure énonciative, elle ne sort donc pas indemne des transformations subies par l'une ou l'autre. La modification énonciativopragmatique, qui vise une simplification du contenu strictement linguistique afin d'agir sur la détermination d'une relation spécifique entre destinateur et destinataire, passe par une modification syntaxique et une modification de la ponctuation.

\subsubsection{Extrait 2}

\section{Extrait avant réécriture (formulaire 1)}

Vous devez aussi fournir une preuve de propriété ou de location du véhicule sous la forme (a) d'une copie du certificat d'enregistrement du véhicule, si vous étiez propriétaire du véhicule, ou (b) d'une copie du contrat de location du véhicule, si vous louiez le véhicule, ou (c) si vous ne possédez plus le certificat d'enregistrement ou le contrat de location, d'une affirmation sous serment que vous étiez propriétaire ou louiez le véhicule en date du 9 septembre 2002. Si (c) s'applique à vous, veuillez utiliser le formulaire d'affidavit joint au présent formulaire de réclamation - cet affidavit doit être signé devant un commissaire à l'assermentation, un avocat ou un officier public (notary public). Si (a) ou (b) s'applique à vous, veuillez envoyer seulement une photocopie du certificat d'enregistrement ou du contrat de location. NE PAS envoyer de documents originaux.

\section{Extrait après réécriture (formulaire 2)}

\section{Documents à fournir}

Avec le présent formulaire rempli et signé, vous devez aussi nous faire parvenir une preuve de propriété ou de location du véhicule en date du 9 septembre 2002.

Les documents mentionnés ci-bas peuvent tous servir de preuve. Cochez le document que vous joindrez à votre formulaire :

$\square$ certificat d'enregistrement du véhicule si vous étiez propriétaire du véhicule 
(une copie et non l'original)

$\square$ contrat de location du véhicule si vous louiez le véhicule (une copie et non l'original)

$\square$ déclaration sous serment (affidavit) que vous étiez propriétaire ou locataire du véhicule si vous ne pouvez fournir l'un ou l'autre des deux premiers documents de cette liste.

Concernant ce nouvel extrait, dans le formulaire 1, il a été choisi de produire un paragraphe compact, constitué par 4 longues phrases. L'une est entièrement en gras permettant ainsi à la fois la respiration de la lecture et la mise en évidence du contenu. Un choix différent a été fait pour le formulaire 2 : la présence de deux paragraphes constitués l'un d'une phrase et le second de deux phrases, sans ponctuation intra-phrastique. La dernière phrase est suivie d'une énumération proposant un choix au lecteur.

\section{Le formulaire 1 :}

- La première phrase comporte 4 virgules : elles servent toutes à articuler les circonstants entre eux.

- La deuxième phrase, en gras, contient 2 virgules : la première sépare la subordonnée circonstancielle de la principale; la seconde sert à une énumération. Par ailleurs, on observe la présence d'un tiret introduisant une explicitation.

- La troisième phrase contient une virgule séparant à nouveau la subordonnée circonstancielle de la principale.

- La quatrième phrase n'a pas de ponctuation intra-phrastique mais la locution négative « NE PAS » est écrite en lettres majuscules afin de souligner l'importance de cette injonction.

Le formulaire 2 : il est constitué de trois phrases. Les deux premières phrases ne contiennent pas de ponctuation intra-phrastique. La troisième comporte une articulation marquée par deux points et introduisant un choix à faire entre trois propositions. La structure visuelle est plus claire que dans le formulaire 1. L'emploi des carrés à cocher (avec retour à la ligne) met l'accent sur la volonté de coénonciation du destinateur avec son destinataire puisque cette ponctuation tend à générer une action de la part du destinataire (effet à la fois pragmatique et sémantique pour la suite de la lecture). Du formulaire 1 au formulaire 2, le rédacteur passe du bien écrire à une écriture efficace.

Dans cet extrait, le R.P. renonce au paragraphe compact du formulaire 1. Il opte pour des phrases plus courtes contenant peu de ponctuation. Lorsque ces phrases contiennent des précisions à apporter, celles-ci sont mises entre parenthèses et ces syntagmes sont non verbaux.

A nouveau, on observe une volonté de clarté (lecture plus fluide, mieux guidée formellement) et de visibilité (compréhension des informations, coopération). On progresse ainsi dans la réception des informations avec des pauses grâce à une disposition qui accompagne le lecteur (forme synthétique et retours à la ligne), contrairement au premier formulaire où les informations sont contenues dans un paragraphe compact sans distinction, à l'exception toutefois d'une phrase entièrement mise en gras.

On constate encore que le R.P. ne supprime pas de phrases (au contraire, il y en a même une de plus). Néanmoins, on regrettera la supression d'informations supposées nécessaires comme «cet affidavit doit être signé devant un commissaire... » et «NE PAS envoyer de documents originaux ». On peut supposer que le R.P. a considéré ces informations comme connues, voire inhérentes implicitement à la démarche. On peut s'interroger sur la pertinence de ce choix. Dans ce second extrait, on aura surtout observé que le choix du R.P. ne s'est pas porté sur une réduction du nombre de phrases, une simplification du contenu, mais sur l'hétérogénéité des signes de ponctuation, avec l'adjonction, aux côtés des points et des virgules, du gras et de la disposition en paragraphes, d'un carré à cocher déterminant la possibilité d'agir du destinataire (retour à la ligne, alinéa marqué par un carré) et d'informations portées entre parenthèses. 


\subsection{Réécriture visant le règlement intérieur de l'accueil enfance d'une mairie}

La seconde transformation que nous allons étudier porte sur la comparaison entre un règlement intérieur de l'accueil enfance d'une commune et sa réécriture sous la forme d'un livret d'accueil destiné aux parents. Comme pour la réécriture du formulaire de réclamation, nous analyserons deux extraits (avant et après réécriture).

\subsubsection{Extrait 1}

Extrait avant réécriture (règlement intérieur)

\section{Article 4: ADMISSION AUX SERVICES EXTRASCOLAIRES, PERISCOLAIRES ET RESERVATION DES PRESTATIONS}

\subsection{Admission}

Le retour des documents suivants est obligatoire pour chaque enfant :

- Dossier unique d'inscription (un par famille).

- La fiche sanitaire (une par enfant).

- Copie du carnet de santé (pages vaccination).

- L'attestation d'assurance scolaire ou d'assurance en responsabilité civile couvrant l'enfant.

- Numéro CAF obligatoire ou à défaut les justificatifs nécessaires à l'établissement du quotient familial (avis d'imposition et dernier relevé de prestations familiales).

- Le cas échéant, une copie des actes de justice portant sur l'exercice de l'autorité parentale.

- Si nécessaire tous documents relatifs à l'état de santé de l'enfant (sous pli fermé).

- Certificat de natation 25 mètres.

\section{Extrait après réécriture (livret d'accueil)}

\section{Inscrire \\ 1. Constituer un dossier d'inscription}

Pour inscrire votre enfant à un ou plusieurs des secteurs cités plus haut (p.3), vous ne devez constituer qu'un seul dossier, à rendre avant la rentrée scolaire de septembre. Ce dernier comportera les documents suivants :

- Le dossier unique d'inscription (un par famille) ;

- La fiche sanitaire (une par enfant).

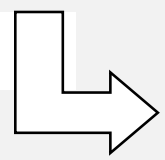

Vous pouvez vous procurer ces deux documents en les retirant à l'Accueil enfance.

Vous devez également joindre à votre dossier :

- Une copie du carnet de santé (pages vaccinations) ;

- L'attestation d'assurance scolaire ou d'assurance en responsabilité civile couvrant l'enfant (pour l'année en cours) ;

- Votre numéro CAF. Si vous ne l'avez pas, vous devez nous fournir une copie de votre avis d'imposition et de votre dernier relevé de prestation familiale ;

- Un certificat de natation 25 mètres de l'enfant ;

- Si nécessaire, tout document relatif à l'état de santé de l'enfant (sous pli fermé). 
On observe ici, tout comme pour le formulaire de réclamation, une différence significative entre les deux extraits, avant et après la réécriture par le R.P.

Le règlement intérieur présente une proposition suivie de deux points annonçant une énumération. Les différents éléments qui suivent sont marqués individuellement par un retour à la ligne et par une puce. Chacun d'eux se clôt par un point. Au total, l'énumération est constituée de 8 syntagmes dont 5 contiennent des informations entre parenthèses.

Le livret d'accueil commence par une phrase comprenant deux circonstants encadrant une principale et séparés par deux virgules. La principale et le second circonstant sont mis en gras.

La deuxième phrase comporte l'énumération de deux documents à fournir (avec retour à la ligne et un tiret). On notera que chaque élément énuméré contient une information mise entre parenthèses.

S'ensuivent deux phrases, séparées par un saut de ligne et un symbole (une flèche) marquant la première. La première ne contient pas de ponctuation intra-phrastique. La seconde présente une articulation marquée par les deux points introduisant une nouvelle énumération de cinq éléments contenant pour trois d'entre eux une information mise entre parenthèses et, pour quatre d'entre eux un groupe de mots mis en gras. Enfin un des éléments de l'énumération est constitué de deux phrases : l'une non verbale et l'autre avec une virgule isolant un circonstant.

La réécriture de ce passage laisse place à une mise en mots plus dense; la forme énumérative est maintenue ainsi que les parenthèses ; le gras fait son apparition ; sur les 4 phrases, une seule contient 2 virgules pour isoler en début et en fin de phrase un circonstant. On voit donc la place qui est faite à la langue et dans le même temps à des outils de ponctuation synthétique comme les parenthèses, mais aussi la mise en gras et la virgule. Toutefois, on observe que le R.P. a fait le choix de supprimer une information «Le cas échéant, une copie des actes de justice portant sur l'exercice de l'autorité parentale » dont on n'est pas certain que, par la suite, elle ne manque pas aux usagers. Ce choix s'avère d'ailleurs discutable en ce qu'il méconnait une volonté de négociation.

Dans ce premier extrait, au final, la réécriture constitue une mise en récit du contenu initial du règlement intérieur, qui reposait exclusivement sur une énumération, et convoque une utilisation variée des signes de ponctuation (points, virgules, points virgules, deux points, tirets, gras, parenthèses, retour à la ligne et paragraphes successifs). Le R.P. recherche donc la création de lien par la coopération linguistique mise en place (sans injonction).

\subsubsection{Extrait 2}

\section{Extrait avant réécriture}

\subsubsection{Accueil de loisirs}

- Accueil de loisirs les mercredis : Les inscriptions ou annulations sont prises jusqu'au vendredi précédent $12 \mathrm{~h} 00$ (dans la limite des places disponibles). Dépassé cet horaire, plus aucune inscription ou annulation ne sera prise en compte.

- Centre de loisirs des vacances : la réservation vacances pour le centre de loisirs ne peut se faire à l'année. Une fiche de réservation (disponible auprès du service enfance ou sur demande par mail) sera à remplir pour chaque période de vacances.

\section{Extrait après réécriture}

Pour l'Accueil Loisirs Sans Hébergement (ALSH enfants et jeunes): 


\author{
Accueil de loisirs les mercredis : vous pouvez inscrire votre enfant soit à l'année, \\ soit occasionnellement. Dans ce dernier cas, l'inscription de votre enfant doit être \\ faite avant le vendredi précédent à midi (dans la limite des places disponibles). Ce \\ même délai s'applique pour toute annulation. Le cas échéant, votre inscription ou \\ votre annulation ne seront pas prises en compte.
}

Accueil de loisirs pendant les vacances : il est impossible de vous y inscrire à l'année. Une fiche de réservation (disponible à l'Accueil enfance ou sur demande par courriel) doit être remplie pour chaque période de vacances.

Dans le règlement intérieur, on observe le recours à deux paragraphes constitutifs d'une énumération contenant un titre en gras avec retour à la ligne et puce : chacun des deux paragraphes énumératifs contient deux phrases et une information mise entre parenthèses. Pour le premier paragraphe, une des deux phrases contient une virgule isolant un circonstant.

Le livret d'accueil présente les informations dans un encadré. Ces informations sont réparties, comme pour le règlement intérieur, en deux paragraphes introduits par un titre en gras suivi de deux points. Le premier paragraphe contient 4 phrases. Si une phrase ne contient pas de ponctuation intra-phrastique, les trois autres contiennent une virgule destinée à une énumération dans l'une, et à isoler un circonstant dans les deux autres (ainsi qu'une information mise entre parenthèses pour l'une de ces deux phrases). Le second paragraphe est constitué, quant à lui, de deux phrases sans ponctuation intra-phrastique à l'exception d'une information entre parenthèses pour l'une des phrases.

A nouveau, dans cette réécriture, on observe une volonté de mise en mots, de produire des phrases supplémentaires convoquant des virgules pour introduire des circonstants ou des énumérations, avec le maintien des parenthèses pour synthétiser des informations supplémentaires/complémentaires.

Finalement, dans ce second extrait, conformément à ce que l'on a relevé pour le premier extrait, on assiste à une volonté de mise en récit du R.P. et à la convocation d'une hétérogénéité de marques de ponctuation participant à la clarté du document, à une structuration spécifique de sa mise en page.

\title{
5 Synthèse
}

Dans le cadre de cet article, nous avons questionné l'aspect communicationnel voire interactionnel de la ponctuation en analysant la réécriture de textes par des R.P. A l'issue de notre analyse, et en convoquant la notion d'intention de communication décrite par Dahlet (2003) qui est mise en place par le scripteur et qui vise à préfigurer des modalités d'interprétation, de sa relation à autrui, nous observons une utilisation spécifique de la ponctuation dans les réécritures analysées.

Si l'on reprend la distinction de Dahlet (2003), conforme à celle d'Anis (1988), entre ponctuation de mots et ponctuation de phrase, on note que c'est la ponctuation du texte lui-même qui est prédominante dans les choix relatifs à la ponctuation lors de la réécriture par le R.P. Plus précisément, on observe que certains signes de séquence (comme l'alinéa, la virgule et le point), certains signes d'énonciation (tels les deux points et les parenthèses) et certains marqueurs expressifs (comme la mise en gras) sont convoqués dans la plupart des extraits réécrits. Les marqueurs les plus fréquents visent donc des faits de segmentation textuelle (cf. Neveu, 2014), autrement dit participent à la structuration matérielle du texte, à la hiérarchisation des localisations, notamment des séquences textuelles (par ex. explication et énumération). On observe ainsi, comme d'autres études l'ont montré dans les dernières années, une diminution lors de la réécriture de la ponctuation interne à l'alinéa permettant une présentation structurée et progressive des informations. La macro ponctuation, en lien avec le contenu langagier, permet donc de mettre en avant des choix notamment d'ordre pragmatique. Ceci permet de faire un lien avec la fonction communicationnelle ou énonciative décrite par Perrot (1980) \& Jaffré (1991), qui associent les choix de ponctuation à ceux de la finalité du message à transmettre. 
On constate une utilisation spécifique de la fonction pragmatique de la ponctuation (à travers le choix du nombre de virgules, mais aussi de parenthèses, des deux points et du point ainsi que du gras, du retour à la ligne/alinéas, des puces ou tirets, ou encore du soulignement) en ce qu'elle se perçoit en dynamique textuelle mais aussi contextuelle (cf. les résultats de Bronckart et al. (1985) et de Schneuwly (1984)). Autrement dit, elle fonctionne simultanément et en interaction avec le choix de la relation mise en place par le R.P. (guidé par le choix du mandataire). Par exemple, dans le formulaire 1 de réclamation et dans le règlement intérieur, où la relation interdiscursive est à la fois injonctive et sur-énonciative, la ponctuation de phrase est quantitativement importante et le contenu compact. Au contraire, lors de la transformation de ces deux documents par des R.P. convoquant une coopération linguistique, on observe soit une ponctuation plus variée dans sa perspective textuelle et non phrastique, soit une simplification du contenu en fonction du texte d'origine.

La relation pragmatique, bien sûr en lien direct avec la syntaxe, commande donc le choix de la ponctuation (de sa répartition plus exactement) :

- si la relation est injonctive/agonale, comme dans le formulaire 1 de réclamation et dans le règlement intérieur, on aura une succession de contenus dans une même phrase et des virgules fréquentes au sein des phrases, mais aussi des énumérations sans accompagnement ;

- si la relation est à visée de coopération/négociation, comme dans le formulaire 2 de réclamation et dans le livret d'accueil, on observera une organisation et une structuration progressive spécifiques de la transmission des informations, mais aussi un choix de simplification (cf. le titre du formulaire de réclamation 2) faisant alterner des phrases avec ponctuation et des phrases sans ponctuation intraphrastique. On notera également le rôle de la mise en relief par le gras, convoqué pour des mots ou groupes de mots, mais aussi le choix des parenthèses qui permet d'éviter de surcharger la phrase en synthétisant le contenu visé à partir de mots clefs. Ce choix peut aussi participer d'une forme de scénarisation injonctive bienveillante, qui n'est pas sans rappeler la mise en relief en gras des productions initiales, visant à prendre la main du lecteur et à l'accompagner sur la route énonciative tracée par le R.P.

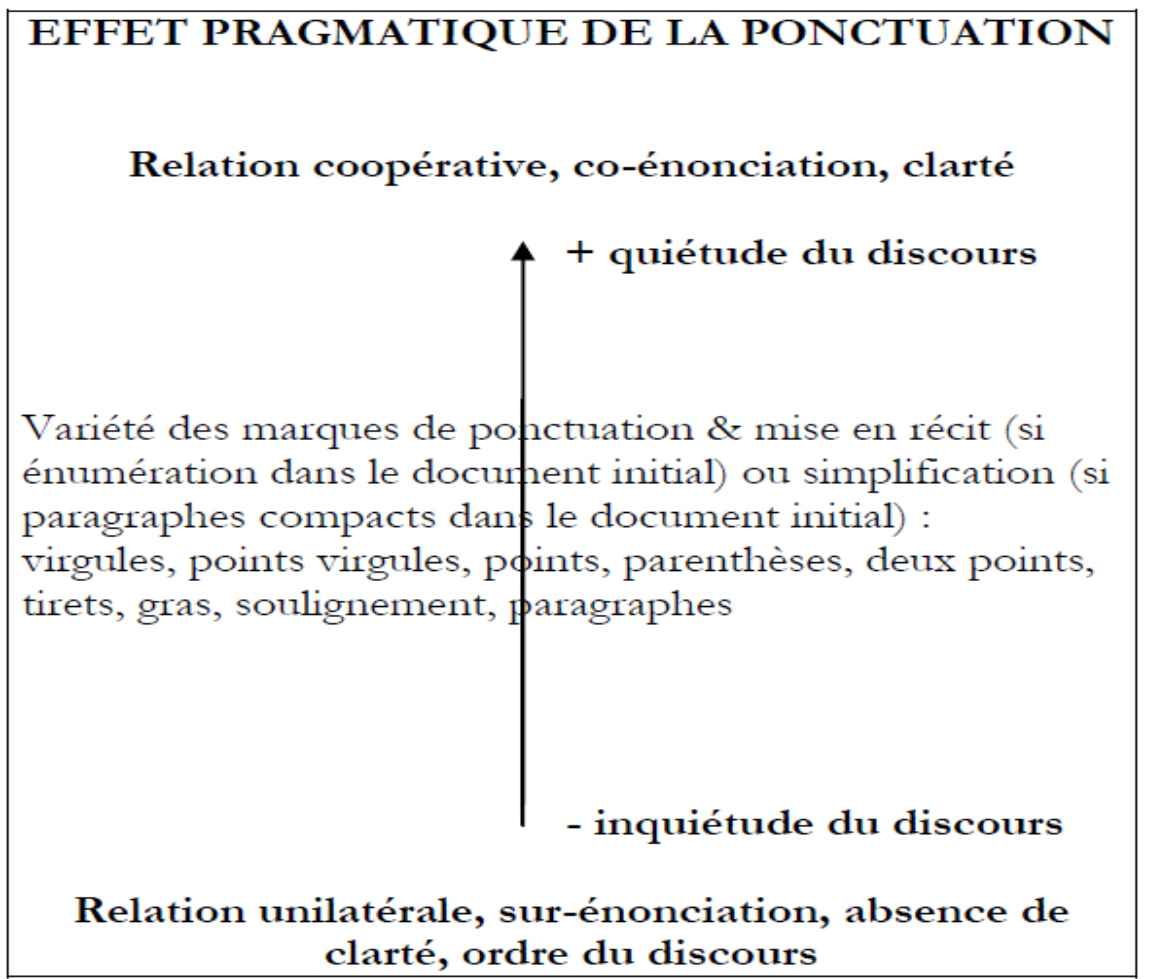


Le R.P. opte pour des phrases contenant peu de ponctuation, des phrases plus courtes lorsque dans la rédaction initiale les phrases et les paragraphes sont compacts, et, au contraire, « réinjecte de la langue », des phrases avec des virgules lorsqu'elles font défaut dans le document initial. Il choisit plus généralement une plus grande variété des marqueurs de ponctuation qui structurent et articulent les différents moments de sa production rédactionnelle et ce dans une perspective inter-phrastique ou textuelle.

L'analyse révèle une spécificité du rédacteur professionnel. Ce dernier ajoute à une pratique certes normalisée de la ponctuation, une dimension pragmatique, intégrant sa représentation du lecteur. Dans sa tâche rédactionnelle, la ponctuation relève donc simultanément de la langue et de la communication en lien avec la relation interdiscursive que le destinateur souhaite mettre en place avec son destinataire. La ponctuation est constitutive d'un espace où se jouent des rapports de places et de faces et une certaine négociation: elle fait partie de ces outils linguistiques contribuant à l'élaboration et à la construction d'une relation voulue spécifique par le R.P. Ces rapports de places et de faces sont donc déterminés mais déterminent aussi l'inquiétude ou la quiétude de l'ordre du discours.

\section{Références bibliographiques}

Anis, J. (2004). Les linguistes français et la ponctuation. In L'Information Grammaticale, N. 102, p. 5-10.

Anis, J., Chiss, J.-L. et Puech, C. (1988). L'Ecriture : théories et descriptions, De Boeck, éditions Universitaires. Arrivé, M., Gadet, F, et Galmiche, M. (1986). La grammaire d'aujourd'hui. Flammarion : Paris.

Beaudet, C. (2010). Pour un enseignement de l'écrit par discipline à l'université. L'autre Forum, Le journal des professeures et professeurs de l'Université de Montréal, La relation professeurs-étudiants aujourd'hui. Virage ou dérives? Vol. 14, no 2, p. 27-29.

Bessonnat, D. (éd.). (1991). Introduction. Pratiques, $n^{\circ} 70$, La ponctuation, p. 85-104.

Bikialo, S. (2004). Le rivage des signes. Remarques sur la ponctuation et l'ailleurs, L'Information grammaticale, $n^{\circ} 102$.

Bronckart, J.-P., Bain, D., Schneuwly, B., Dayaud, C. et Pasquier A. (1985). Le fonctionnement des discours. Neuchâtel : Delachaux et Niestlé.

Catach, N. (1980). Présentation. Langue française. $N^{\circ} 45$, La ponctuation, p. 16-27.

Clerc, I. \& Beaudet, C. (éds.). (2008). Langue, médiation et efficacité communicationnelle. Québec : Presses de l’Université Laval.

Dahlet, V. (2003). Ponctuation et énonciation. Ibis Rouge.

Drillon, J. (1991). Traité de la ponctuation française. Paris : Gallimard.

Dürrenmatt, J. (1998). Bien coupé mal cousu. De la ponctuation et de la division du texte romantique. PUV.

Fayol, M. (1988). Une approche psycholinguistique de la ponctuation. Etude en production et en compréhension. Langue Française, $n^{\circ} 81$.

Fayol, M. et Abdi, H. (1988). Influence of script structure on ponctuation. European Bulletin of Cognitive Psychology, $n^{\circ} 8$.

Fayol, M. et Lété, B. (1987). Ponctuation et connecteurs : une approche textuelle génétique. European Journal of Psychology of Education, $n^{\circ} 2$.

Hayes, J.R. et Flower, L.S. (1980). Identifying the organization of writing process. In L.W. Gregg et E.R. Steinberg (éds), Cognitive Process in writing. Hillsdale : Erlbaum.

Jaffré, J.-P. (1991). La ponctuation du français : études linguistiques contemporaines. In D. Bessonnat (éd.), Pratiques, $n^{\circ} 70$, La ponctuation, p. 61-83.

Jagueneau, L. (2014). La ponctuation dans un corpus épistolaire ordinaire. Revue La Licorne, Numéro 52 (ÉPUISÉ). En ligne : http://licorne.edel.univ-poitiers.fr/document5882.php (consulté le 19/04/2014).

Labasse, B. (2008). Modeling the communication of complexity in an information-saturated

society. In C. Beaudet, P. Grant-Russel \& D. Starke Meyerring (éds.), Research Communication in the Social and Human Sciences: from dissemination to public engagement (pp.60-84). UK : Cambridge Publishing Press.

Lapacherie, J.-G. (2014). De quoi les «signes de ponctuation » sont-ils les signes ? Revue La Licorne, Numéro 52 (ÉPUISÉ) . En ligne : http://licorne.edel.univ-poitiers.fr/document5705.php (consulté le 19/04/2014).

Leblanc, J. (1998). La ponctuation face à la théorie de l'énonciation. In J.-M. Defays, L. Rosier et F. Tilkin (éds), A qui appartient la ponctuation? (p.97-98) Paris : Duculot, coll. Champs linguistiques.

Narjoux, C. (2010). La ponctuation. Règles, exercices et corrigés. Bruxelles : De Boeck Duculot.

Neveu, F. (2014). De la syntaxe à l'image textuelle ponctuation et niveaux d'analyse Linguistique. Revue La 
Licorne, Numéro 52 (ÉPUISÉ). En ligne : http://licorne.edel.univ-poitiers.fr/document5688.php (consulté le 19/04/2014).

Orlandi, E. (2007). Un point c'est tout. In Figures d'ajout. Phrase, texte, écriture, Presses Sorbonne Nouvelle.

Perrot, J. (1980). Ponctuation et fonctions linguistiques. In Langue française. $N^{\circ} 45$, La ponctuation. p. 67-76.

Passerault J.-M. (1991). La ponctuation. Recherches en psychologie Du langage. In D. Bessonnat (éd.), Pratiques, $n^{\circ} 70$, La Ponctuation, p. 85-104.

Pétillon, S. (2004). Présentation. L'Information Grammaticale, $N^{\circ} 102$, p. 3-4

Popin, J., (1998). La ponctuation. Paris : Nathan.

Pereira, M.-E., Romain, C. et Rey, V. (2015). «L'écriture professionnelle : un bel enjeu didactique au croisement de la littérature, de la rhétorique et de la linguistique ». In Beaudet C. \& Rey V. Ecritures expertes en questions (pp. 253-263) Aix-en-Provence : Presses Universitaires de Provence.

Romain, C., Pereira, M.-E. et Rey, V. (2015a). Enjeux de politesse interactionnelle et de coopération dans des écrits fonctionnels ou des écrits professionnels : étude de cas. In C. Beaudet et V. Rey (éds.), Ecritures expertes en questions (pp. 73-86). Aix-en-Provence : Presses Universitaires de Provence.

Romain, C., Pereira, M.-E. et Rey, V. (2015b). Ecriture professionnelle et contexte de tension : la répétition, un outil d'orientation co-énonciatif du lecteur. Le discours et la langue - Revue de linguistique française et d'analyse du discours, $n^{\circ} 7-2, p .79-96$.

Saint-Gérand, J.-P. (2000) [mis en ligne 2014]. Un point, c'est tout... : grammaires, dictionnaires, et poétique du langage entre XIXe et XXe siècles, Revue La Licorne, $N^{\circ} 52$, La ponctuation, Presses Universitaires de Rennes, http://licorne.edel.univ-poitiers.fr/document5703.php.

Schneuwly, B. (1984). Le texte discursif écrit à l'école. Thèse pour le doctorat, Université de Genève.

Tournier, C. (1980). Histoire des idées sur la ponctuation, des débuts de l'imprimerie à nos jours. Langue française. $N^{\circ} 45$, La ponctuation, p. 28-40.

Védénina, L.G. (1989). Pertinencelinguistique de la présentation typographique. Peeters/Selaf.

Van Dijk, T.A. et Kintsch, W. (1983). Stategies of discourse comprehension, New York: Academic Press.

\footnotetext{
${ }^{1}$ Voici les exemples qu'il cite p.75:

«-Banque populaire clients offrir impôt service etc.

-La Banque Populaire vous offre l'impôt service pour en finir avec les impôts qui tombent au mauvais moment.

-Pour en finir avec les impôts qui tombent au mauvais moment, la Banque Populaire vous offre l'impôt service. »

2 «Les signes de démarcation (blanc typographique directement lié au sens ; majuscules et points qui limitent la phrase ; virgule qui aide le lecteur à s'orienter dans le texte) font apparaître des oppositions significatives [31], [31']. Les signes de régulation font entrer les membres d'une phrase dans tel ou tel segment et permettent au sujet d'inclure (ou non) un membre dans le noyau qui véhicule l'information essentielle [32], [32'].

Les signes de qualification regroupent des signes à valeur modale (les guillemets, pour un changement de registre

[33] ; les points d'interrogation et d'exclamation, pour une modalité non assertive [34]).

[31] La fête battait son plein, mais les veilleurs restaient à guetter dans leur cachette.

[31'] La fête son plein. Mais les veilleurs restaient à guetter dans leur cachette.

[32] Il a mangé beaucoup de salade au déjeuner.

[32'] Il a mangé beaucoup de salade, au déjeuner.

[33] « Sophie », c'est le camion.

[34] « La clef, celle d'en haut !»
}

${ }^{3}$ cf. Julie Leblanc (1998 : 87) mais aussi Jacques Popin (1998: 100) qui parle de signes par «évocation ». 